\title{
Behaviour of a sperm surface transmembrane glycoprotein basigin during epididymal maturation and its role in fertilization in mice
}

\author{
D. K. Saxena ${ }^{1}$, T. Oh-oka ${ }^{1}$, K. Kadomatsu ${ }^{2}$, \\ T. Muramatsu ${ }^{2}$ and K. Toshimori ${ }^{*}$ \\ ${ }^{1}$ Department of Anatomy and Reproductive Cell Biology, Miyazaki Medical College, \\ Kihara 5200, Kiyotake, Miyazaki 889-1692, Japan; and 2 Department of Biochemistry, \\ Nagoya University School of Medicine, Nagoya, Japan
}

\begin{abstract}
Basigin (bsg) is a transmembrane glycoprotein belonging to an immunoglobulin superfamily and is localized on the surface of the sperm tail. The behaviour of bsg during epididymal maturation and its role in fertilization were examined using an anti-bsg antibody. Spermatozoa from caput, corpus and cauda epididymides were immunostained by indirect immunofluorescence (IIF). Immunostaining revealed that bsg is localized on the principal piece of caput spermatozoa and the molecule was found on the middle piece during transit in the corpus and cauda epididymides. Concomitantly, the molecular mass of bsg was reduced from $37 \mathrm{kDa}$ (testis) to $26 \mathrm{kDa}$ (cauda epididymidis). IVF experiments were designed to assess the effect of anti-bsg antibody on the fertilization events. Anti-
\end{abstract}

bsg antibody significantly inhibited primary binding to the cumulus-invested oocytes with intact zonae pellucidae in a dose-dependent manner. Consequently, the fertilization rate of cumulus-invested oocytes with intact zonae pellucidae was also inhibited. The bsg molecule was also detected on the head of live capacitated spermatozoa by IIF under IVF conditions. These findings indicate that testicular bsg is a glycosylated protein that undergoes molecular processing and deglycosylation during its transit in the epididymis. The bsg molecule that was detected on the sperm head after capacitation may facilitate the primary binding or might be involved in distinct events required for primary binding of spermatozoa to the zona pellucida during capacitation and sperm-cumulus interaction.

\section{Introduction}

Basigin (bsg) is a transmembrane glycoprotein that belongs to the immunoglobulin superfamily. The mouse bsg molecule is similar (in terms of DNA sequence) to other transmembrane glycoproteins of the immunoglobulin superfamily, such as human bsg and EMMPRIN (CE9/MC31 or M6). These molecules have been implicated in the activation of lymphocytes, as well as in the induction of matrix metalloproteinase (Kasinrerk et al., 1992; Biswas et al., 1995), and in neuronal-glial interaction in the retina during development (Fadool and Linser, 1993). In addition, bsg is essential for fertility in both males and females (Kuno et al., 1998). In males, it is essential for the completion of spermatogenesis, as supported by the observation that bsg null mice are azoospermic (Igakura et al., 1998), whereas in females, bsg is required for maintaining normal reproductive functions, as supported by the findings that bsg null female mice show multiple reproductive failure including ovulation and implantation (Igakura et al., 1998; Kuno et al., 1998). Bsg is expressed differently during mouse embryogenesis (Fan

*Correspondence

Email: ktoshi@post.miyazaki-med.ac.jp et al., 1998) and its expression can be followed by its glycosylation pattern.

In terms of molecular structure, the extracellular domain of bsg contains two randomly arranged Ig domains, Ig-like C2 domains and Ig-like V-domains (Miyauchi et al., 1990, 1991). Bsg is a highly glycosylated membrane protein with a molecular mass of 43-66 kDa. Glycosylation varies depending on the type of tissue (Nehme et al., 1995; Igakura et al., 1998). N-terminal IgG type domains of the bsg molecule are involved in homo-oligomer formation on the plasma membrane of the testes (Yoshida et al., 2000), and this may have functional importance.

It has been suggested that the bsg molecule may be involved in cell-to-cell interaction and have functional significance in various biological functions (Kuno et al., 1998; Yoshida et al., 2000). Transmembrane glycoprotein molecules, such as EMMPRIN, CE9 and HT7, have been reported to be integrin-associated (Berditchevski et al., 1997). Several integrin-associated molecules are involved in adhesion and cell-to-cell interactions, including the fusion of gametes (for a review, see Evans, 1999). The fundamental mechanisms of adhesion and intercellular interactions are well conserved in all cells irrespective of their origin (somatic or reproductive).

The bsg molecule is expressed in the testis and localized 
on the principal piece of testicular spermatozoa (D. K. Saxena and K. Toshimori, unpublished). The redistribution of sperm surface, domain-specific protein CE9, has been reported during epididymal maturation in rats (Nehme et al., 1993). Another sperm surface molecule, MC31, is similar (identical DNA sequence) to CE9 (Wakayama et al., 2000), and also shows similar redistribution during epididymal maturation in rats (Toshimori, 2000). Both CE9 and MC31 are also expressed in the testis and on the principal piece of testicular spermatozoa. These molecules show partial homology to the bsg molecule. The involvement of bsg in spermatogenesis has been reported on the basis of the findings in bsg null mice. However, the molecular nature of the testicular and sperm bsg molecule, such as glycosylation and molecular changes, if any, during epididymal transit remains to be investigated. In addition, the role of bsg during fertilization has not been evaluated, because knockout mice are azoospermic.

Hence, it is of interest to gain a better understanding of the molecular nature of the testicular and sperm bsg, its modifications and redistribution, if any, during epididymal transit, and its role during various events of fertilization. With this background, the behaviour of the bsg molecule during epididymal transit and its role during fertilization were examined using a specific rabbit anti-bsg antibody.

\section{Materials and Methods}

\section{Animals}

ICR mice at 3 months of age were obtained from Kyudo Company (Kumamoto) and maintained thereafter in the laboratory animal centre under the animal welfare guidelines of our college. The animals were anaesthetized with diethyl ether and killed by cervical dislocation.

\section{Reagents, IVF culture medium and antibodies}

All chemicals used in this study were of analytical and culture grade, and were obtained from Nacalai Tesque Inc. (Kyoto) unless otherwise stated. A modified Krebs'-Ringer bicarbonate solution (TYH medium; Toyoda et al., 1971) was used for gamete manipulation and fertilization throughout the study. The protein (antibody) concentration was estimated by the bicinchonic acid (BCA) method according to the supplier's manual (Pierce Chemical Co., Rockford, IL).

Purified rabbit anti-bsg antibody lgG raised against a glutathione S-transferase (GST)-bsg fusion protein containing the entire extracellular domain of mouse bsg (Fan et al., 1998) was used as a probe. Another monoclonal antibody mMC31 (IgG) recognizes MC31, a sperm surface molecule, and has a high degree of similarity to bsg on sperm flagella. This anti-MC31 antibody does not recognize mice spermatozoa (Toshimori et al., 1992) and does not affect fertilization in mice (Saxena et al., 1999). Hence, mMC31 (an irrelevant $\operatorname{lgG}$ ) was used as control throughout the study. Pre-immune rabbit serum (rabbit $\lg G$ ) was also used as the control for IVF-IIF.
Antibody mMC31 was purified according to a method described by Toshimori et al. (1998) using a Protein A column (Ampure PA kit; Amersham International, Little Chalfont). The peak fraction was desalted with a TYH medium pre-equilibrated Sephadex G 25M column (PD-10 column) to exchange binding buffer with TYH medium. The resulting purified antibody $\left(2.0-3.7 \mu \mathrm{g} \mathrm{ml}^{-1}\right.$ peak yield) was stored at $-80^{\circ} \mathrm{C}$ until used for the IVF study.

\section{Determination of bsg molecular mass by western blotting}

Spermatozoa were collected by mincing testis and caput, corpus and cauda epididymides with scissors in PBS. The tissues were then washed three times with 0.1 mol PBS I-1 and centrifuged at $450 \mathrm{~g}$ for $5 \mathrm{~min}$. The samples were extracted with $0.1 \%$ (v/v) Triton-X100 in 0.1 mol PBS I-1 containing proteinase inhibitors, $0.2 \mathrm{mmol}$ phenylmethylsulphonyl fluoride $\mathrm{I}^{-1}$ (Sigma, St Louis, MO), $1.0 \mu \mathrm{g}$ pepstatin $\mathrm{A} \mathrm{ml}^{-1}$ and $1.0 \mu \mathrm{g}$ leupeptin $\mathrm{ml}^{-1}$ (Nacalai Tesque, Kyoto) for $30 \mathrm{~min}$ at $0^{\circ} \mathrm{C}$ with shaking. After centrifugation at $20000 \mathrm{~g}$ for $30 \mathrm{~min}$, the supernatant was precipitated with ice-cold acetone. The extract was dissolved in a sample buffer consisting of $1 \%(\mathrm{w} / \mathrm{v})$ SDS, $1.0 \mathrm{mmol}$ phenylmethylsulphonyl fluoride $\mathrm{I}^{-1}$, 20\% (v/v) glycerol, $62.5 \mathrm{mmol}$ Tris- $\mathrm{HCl} \mathrm{I}^{-1}$ and bromophenol blue. The samples were then analysed by SDS-PAGE according to the procedure of Laemmli (1970) using a 10\% (w/v) polyacrylamide gel. Western blot analysis was carried out according to the method described by Towbin et al. (1979). The blot was immunostained using the anti-bsg antibody as the primary antibody and horseradish peroxide-conjugated anti-rabbit IgG (Protos Immunoresearch, Burlingame, CA) as the secondary antibody.

\section{Localization of bsg on caput, corpus and cauda epididymal (during epididymal maturation) and capacitated spermatozoa fixed in paraformaldehyde by indirect immunofluorescence (IIF assay)}

Isolated caput, corpus and cauda epididymal and capacitated spermatozoa were fixed in $1 \%(\mathrm{w} / \mathrm{v})$ paraformaldehyde for $20 \mathrm{~min}$. Fixed spermatozoa were washed three

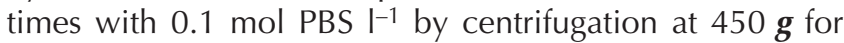
$5 \mathrm{~min}$ and then incubated for $1 \mathrm{~h}$ with the anti-bsg antibody (at a dilution of 1:40). After incubation for $1 \mathrm{~h}$, these spermatozoa were washed three times and incubated with Alexa 488 (anti-rabbit IgG; Molecular Probes, Eugine, OR) as a secondary antibody (at a dilution of 1:300) for $30 \mathrm{~min}$, and then washing was repeated. Finally, sperm samples were placed on a slide under a coverslip and examined under a fluorescence apparatus-equipped light microscope (BX-50 type; Olympus; Tokyo). Both immunofluorescent images and the corresponding differential interference contrast (DIC) images were observed.

\section{Immunocytochemistry of the oocyte}

Immunostaining of oocytes was performed in the same way as described above, except that the oocytes were 
washed in fresh PBS three times. This procedure was carried out to determine whether the anti-bsg antibody would crossreact with oocyte components.

\section{Immunocytochemical localization of bsg on live capacitated spermatozoa by indirect immuno- fluorescence assay under IVF conditions (IVF-IIF assay)}

The immunostaining method was modified to determine the behaviour of bsg on live spermatozoa undergoing capacitation under IVF conditions. Spermatozoa were incubated in $\mathrm{TYH}$ medium for $1.5 \mathrm{~h}$ at $37^{\circ} \mathrm{C}$ for capacitation as described earlier. After incubation for $1.5 \mathrm{~h}$, anti-bsg antibody (at 1:40 concentration) was added to the capacitation medium before being incubated for $1 \mathrm{~h}$. These spermatozoa were transferred to fresh TYH medium and washed three times in TYH media by transfer washing. The resulting spermatozoa were then incubated with Alexa 488 (antirabbit IgG) as the secondary antibody (at 1:40 concentration in $\mathrm{TYH}$ ) for $30 \mathrm{~min}$ at $37^{\circ} \mathrm{C}$. The sperm samples were

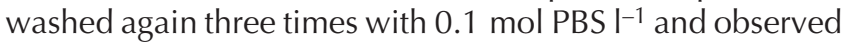
under a fluorescence apparatus-equipped light microscope (BX-50).

Capacitated spermatozoa were double-labelled with $\mathrm{mMN7}$ by routine IIF as described above using mouse IgG $(\mathrm{H}+\mathrm{L})$-Rhodamine (TAGO Inc., Burlingame, CA) as secondary antibody. This procedure was carried out to assess the status of the acrosome, as mMN7 recognizes an intraacrosomal antigen molecule 'acrin 1' (Saxena et al., 1999). Images were obtained using the green channel, the red channel and DIC using Coolsnap digital video-PC system (Roper Scientific, Acton, MA) equipped with a light microscope (BX-50 type). Images were processed further with Adobe Photoshop software (Adobe system Inc., Mountain View, CA). Acrosome-reacted spermatozoa (the zona pellucida-induced) were also immunolabelled using the same method.

\section{IVF experiments}

IVF experiments were conducted to assess the effect of the anti-bsg antibody on fertilization events. Superovulation was induced in female mice ( 9 weeks) by consecutive i.p. injections of 5 iu equine chorionic gonadotrophin (eCG) and 5 iu hCG $48 \mathrm{~h}$ later. Ovulated egg masses were collected from oviducts $15 \mathrm{~h}$ after hCG administration. The cumulus-oocyte complexes were released by rupturing the oviductal ampullae and were immediately pulled into the TYH medium, which had been equilibrated with $5 \% \mathrm{CO}_{2}$ in air under oil (dimethyl polysiloxane; Sigma). The cumulusoocyte complexes were treated briefly with $0.01 \%(\mathrm{w} / \mathrm{v})$ hyaluronidase in TYH medium to remove cumulus cells and to prepare the cumulus-free oocytes. The zona pellucidafree oocytes were prepared by briefly incubating the cumulus-free oocytes with TYH medium at a low $\mathrm{pH}$ ( $\mathrm{pH} 2.5$; the modified Krebs'-Ringer bicarbonate solution), as described previously (Saxena et al., 1999).

Spermatozoa were collected from the distal portion of cauda epididymidis of mature male mice using a blade. The spermatozoa were allowed to disperse into $400 \mu \mathrm{l}$ TYH medium under oil in an IVF dish, before being diluted to a final concentration of $2 \times 10^{5}$ spermatozoa $\mathrm{ml}^{-1}$ in TYH medium and then incubated for $2 \mathrm{~h}$ at $37^{\circ} \mathrm{C}$ in $5 \% \mathrm{CO}_{2}$ for capacitation. Capacitated spermatozoa were co-incubated with zona pellucida proteins to induce an acrosome reaction. Collection of the zona pellucida proteins and induction of the acrosome reaction by these proteins were performed in accordance with the methods described by Saxena et al. (1999).

Capacitated spermatozoa were used to inseminate the cumulus invested-oocytes (zona pellucida intact) in TYH medium containing anti-bsg antibody at 0 (control), 50 or $100 \mu \mathrm{g} \mathrm{ml}^{-1}$ and $\mathrm{mMC} 31$ antibody at $100 \mu \mathrm{g} \mathrm{ml}-1$. These experiments were carried out to determine whether anti-bsg antibody affects sperm-zona pellucida interaction and fertilization.

The acrosome-reacted spermatozoa inseminated the zona pellucida-free oocyte in TYH medium containing antibsg antibody at 0.0 (control) or $100 \mu \mathrm{g} \mathrm{m}^{-1}$ and mMC31 antibody at $100 \mu \mathrm{g} \mathrm{ml}^{-1}$. These experiments were carried out to determine whether anti-bsg antibody affects spermoocyte binding and fusion. At least 50 oocytes in each group were observed. The final concentration of spermatozoa was adjusted to approximately $4 \times 10^{4}$ spermatozoa $\mathrm{ml}^{-1}$ in a $400 \mu \mathrm{l}$ droplet of TYH medium.

\section{Assessment of IVF events}

The percentage of motile spermatozoa was estimated by the hanging drop preparation method (Mastroianni and Manson, 1963). Sperm motility and fertilization events, such as sperm binding and pronucleus formation, were monitored under an inverted microscope (IM type; Olympus) through a charge-coupled device (CCD) camera (Model KP-M1; Hitachi Denshi Ltd, Tokyo) for $30 \mathrm{~min}$, at both $1 \mathrm{~h}$ and $5 \mathrm{~h}$ after insemination.

Primary sperm binding. Sperm-oocyte complexes from the cumulus-free oocytes and the cumulus-invested oocyte groups were transferred to a drop of fresh media at $30 \mathrm{~min}$ and $1 \mathrm{~h}$ after insemination, respectively. These samples were incubated for 10-30 min, and then fixed in a drop of $4 \%(\mathrm{w} / \mathrm{v})$ paraformaldehyde. During these steps, most of the non-specifically attached spermatozoa detached from the zona pellucida. These sperm-oocyte complexes were transferred to a droplet of fresh medium, and spermatozoa bound at the focal plane of the maximum diameter of the zona pellucida were counted under an inverted phase-contrast microscope (Olympus), and photographed. A minimum of 50 oocytes was examined in each group.

\section{Fertilization}

The fertilization rates of both the zona pellucida-intact and the zona pellucida-free oocytes were determined under a phase-contrast microscope by the presence of pronuclei 
Table 1. Effect of anti-basigin (bsg) antibody on primary binding of spermatozoa to the zona pellucida in mice

\begin{tabular}{|c|c|c|c|c|}
\hline \multirow[b]{3}{*}{ Treatment } & \multicolumn{4}{|c|}{ Number of spermatozoa bound per oocyte (mean \pm SD) } \\
\hline & \multicolumn{2}{|c|}{ Cumulus-intact oocytes } & \multicolumn{2}{|c|}{ Cumulus-free oocytes } \\
\hline & $\begin{array}{c}\text { Number of } \\
\text { oocytes observed }\end{array}$ & Primary binding* & $\begin{array}{c}\text { Number of } \\
\text { oocytes observed }\end{array}$ & Primary binding ${ }^{\dagger}$ \\
\hline Anti-bsg & 65 & $2.9 \pm 1.2^{\mathrm{a}}$ & 48 & $9.2 \pm 0.9^{d}$ \\
\hline TYH (control) & 55 & $13.2 \pm 2.1^{b}$ & 35 & $15.0 \pm 0.9_{f}^{\mathrm{e}}$ \\
\hline mMC31 (control) & 42 & $14.8 \pm 2.0^{\mathrm{C}}$ & 32 & $13.8 \pm 3.5^{f}$ \\
\hline
\end{tabular}

Experiments were repeated three times.

a versus $b$ and $c$, and $d$ versus e and f: $P<0.001$.

* Observation was done after transfer washing at $1.5 \mathrm{~h}$.

${ }^{+}$Observation was done after transfer washing at $0.5 \mathrm{~h}$.

Table 2. Effect of anti-basigin (bsg) antibody on fertilization of zona-intact oocytes of mice

\begin{tabular}{lccc}
\hline Treatment & $\begin{array}{c}\text { Antibody } \\
\left(\mathrm{mg} \mathrm{ml}^{-1}\right)\end{array}$ & $\begin{array}{c}\text { Total number of } \\
\text { oocytes observed }\end{array}$ & $\begin{array}{c}\text { Percentage of pronucleus } \\
\text { formation (mean } \pm \mathrm{SD})\end{array}$ \\
\hline Anti-bsg & 0.10 & 80 & $20.2 \pm 4.4^{\mathrm{a}}$ \\
TYH (control) & 0.05 & 38 & $53.6 \pm 8.2^{\mathrm{b}}$ \\
mMC31 (control) & 0 & 75 & $76.7 \pm 8.1^{\mathrm{c}}$ \\
\hline
\end{tabular}

Experiments were repeated four times.

$\mathrm{a}$ and $\mathrm{b}$ versus $\mathrm{c}$ and $\mathrm{d}: P<0.001$.

at $5 \mathrm{~h}$ after or at the two-cell stage at $24 \mathrm{~h}$ after the coincubation of gametes.

\section{Statistical analyses}

Each experiment was repeated three to five times. The data were analysed using the Student's $t$ test (Table 1) and chi-squared test (Table 2). $P<0.05$ was considered to be significant.

\section{Results}

Immunocytochemical localization of bsg on caput, corpus and cauda epididymal spermatozoa, and on capacitated spermatozoa by IIF

Immunostaining of fixed testicular and epididymal spermatozoa from caput, corpus and cauda epididymides revealed that bsg is localized on the principal piece of $>74 \pm 7.0 \%$ of testicular and $75 \pm 4.6 \%$ of caput spermatozoa. The bsg molecule was found on the middle piece during epididymal transit in the corpus $(62 \pm 2.5 \%)$ and caudal epididymides (78 $\pm 2.5 \%$ ). Two hundred spermatozoa (fixed in paraformaldehyde) were examined in each group (Fig. 1a-h).

After capacitation, bsg remained localized on the middle piece, showing stronger staining on approximately $77 \pm 14 \%$ of 200 capacitated spermatozoa (fixed in paraformaldehyde) examined (not shown). Only $12 \pm 0.5 \%$ of fixed capacitated spermatozoa showed staining of the head, predominantly at the apical region of the anterior acrosome. Staining was observed on $<3 \%$ of spermatozoa (including head and tail components) in control groups treated with mMC31, preimmune rabbit serum or only the secondary antibody representing non-specific staining.

\section{Immunocytochemical localization of bsg on live capacitated spermatozoa by modified IIF assay (under IVF condition IVF-IIF assay)}

The percentage of live capacitated spermatozoa with a strongly stained middle piece was $73 \pm 2.6 \%$ and for the head was $21 \pm 1.5 \%$, including the head and middle piece as shown (Fig. 2a-d). The percentage of spermatozoa showing any staining (including head or tail components) was $<5 \%$ in control groups treated with mMC31, preimmune rabbit serum and only secondary antibody under living conditions.

Acrosomal status determined by tracing the immunocytochemistry images for an intra-acrosomal antigen, acrin 1, with pertinent antibody, mMN7 (Fig. 2a-d) revealed that spermatozoa with staining of the apical resin in the head region are also immunostained with mMN7 and are acrosome intact.

Acrosome-reacted spermatozoa were also immuno- 

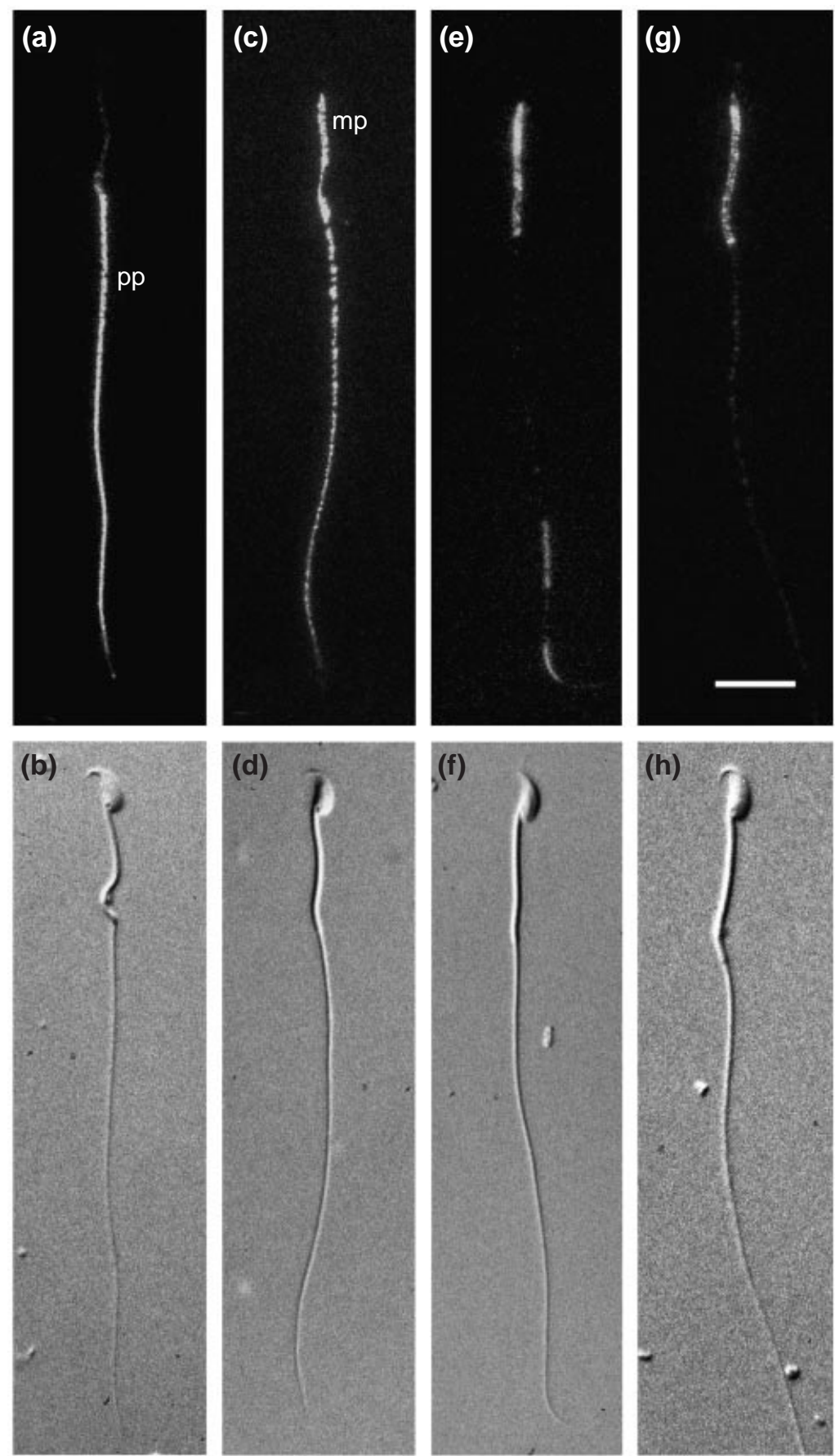

Fig. 1. Immunofluorescence localization of the basigin molecule on spermatozoa from mouse epididymis by indirect immunofluorescence assay. (a,b) Caput, $(\mathrm{c}, \mathrm{d})$ corpus and $(\mathrm{e}, \mathrm{f})$ cauda epididymal spermatozoa, and $(\mathrm{g}, \mathrm{h})$ capacitated spermatozoa. (a,c,e,g) Immunofluorescent images and (b,d,f,h) the corresponding differential interference contrast images. Note immunostaining changes during epididymal transit. Staining is found on the principal piece (pp) of spermatozoa at the caput epididymides $(a, b)$, on both the middle piece $(\mathrm{mp})$ and the principal piece at the corpus epididymides $(\mathrm{c}, \mathrm{d})$, on the middle piece with some remaining immunostaining on the principal piece region in the cauda epididymides $(e, f)$ and, typically, only on the middle piece region in capacitated spermatozoa (g,h). Scale bar represents $11.2 \mu \mathrm{m}$. 

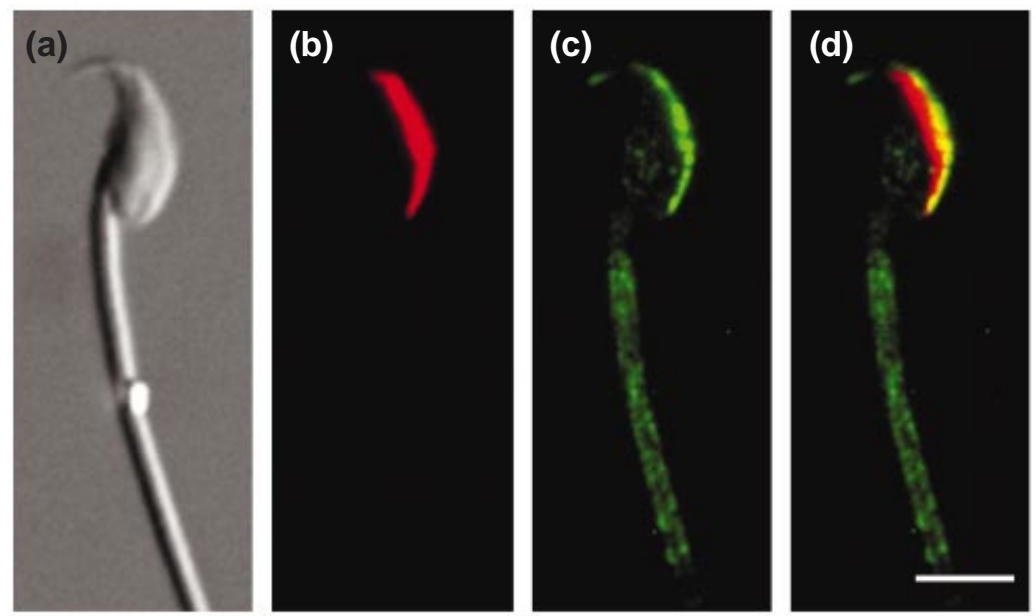

Fig. 2. Immunocytochemical localization of the basigin molecule on live capacitated spermatozoa of mice by indirect immunofluorescence assay under IVF conditions (IVF-indirect immunofluorescence assay). (a) Differential interference contrast image; (b) immunofluorescence image of acrin 1 (red), which is obtained using red channel for intra-acrosomal antigen acrin 1; (c) immunofluorescence image of basigin (green), which is obtained using green channel; (d) merged images (yellow) of acrin 1 (red) and basign (green). Basigin was observed at the mid-piece region and expressed strongly at the apical region of the acrosome. Scale bar represents $2 \mu \mathrm{m}$.
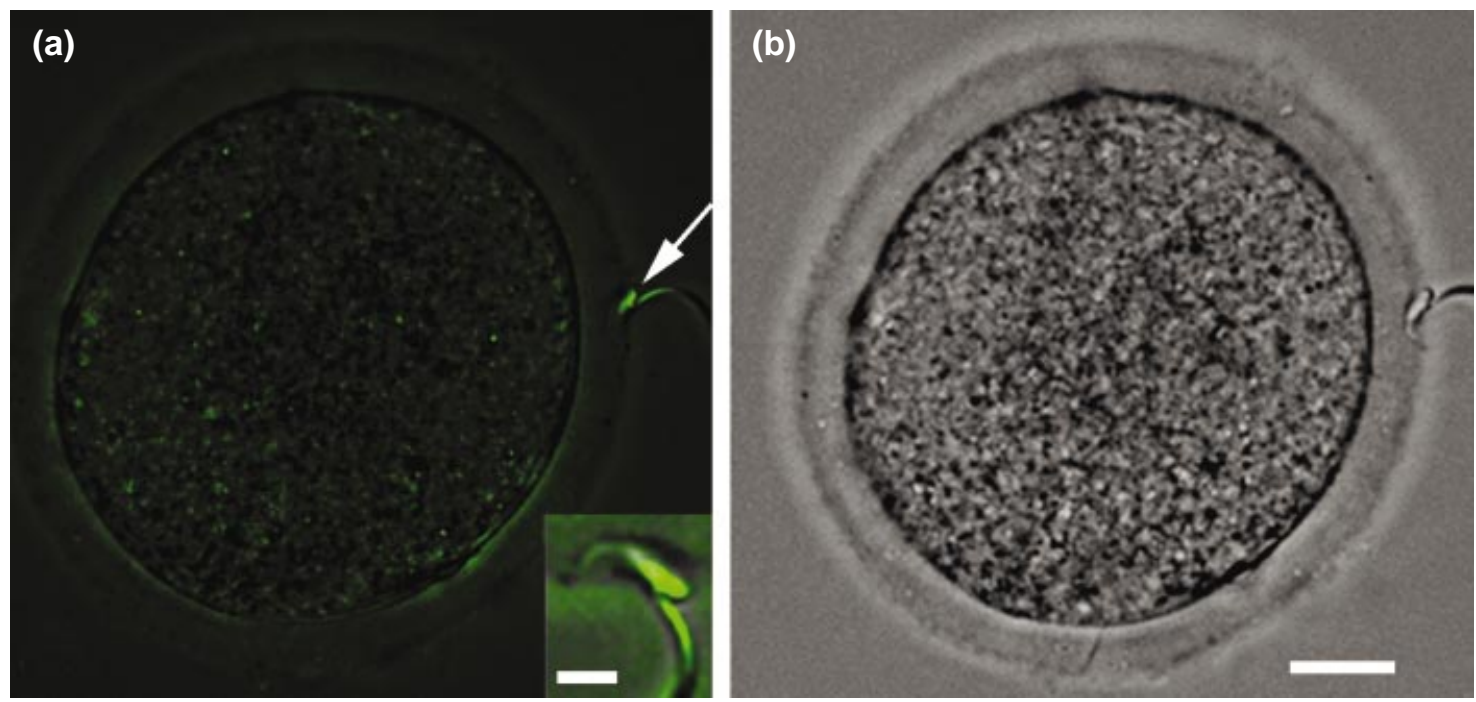

Fig. 3. Photographs showing the presence of basigin at the postacrosomal region and the middle piece of a mouse spermatozoon bound to the zona pellucida (arrow and inset) by indirect immunofluorescence assay in the presence of anti-basigin antibody. This oocyte was recovered $1 \mathrm{~h}$ after insemination. Note no immunostaining was observed on the zona pellucida. (a) Immunofluorescence image and (b) differential interference contrast image. Scale bars represent (a,b) $22 \mu \mathrm{m}$ and (inset) $1.6 \mu \mathrm{m}$.

stained with anti-bsg antibody. As a result, the bsg molecule was detected on the postacrosomal resin (Fig. 3) and also at the equatorial segment of a few acrosome-reacted spermatozoa (Fig. 4). These spermatozoa were negatively immunostained for an intra-acrosomal antigen, acrin 1, by IIF using mMN7. Negative staining for this antigen indicates completion of the acrosome reaction.

\section{Immunostaining of sperm-oocyte complexes by IIF}

Immunostaining of sperm-oocyte complexes was performed to examine crossreactivity of anti-bsg antibody with oocyte components. As a result, anti-bsg antibody did not label any component of oocyte or the zona pellucida (not shown). 

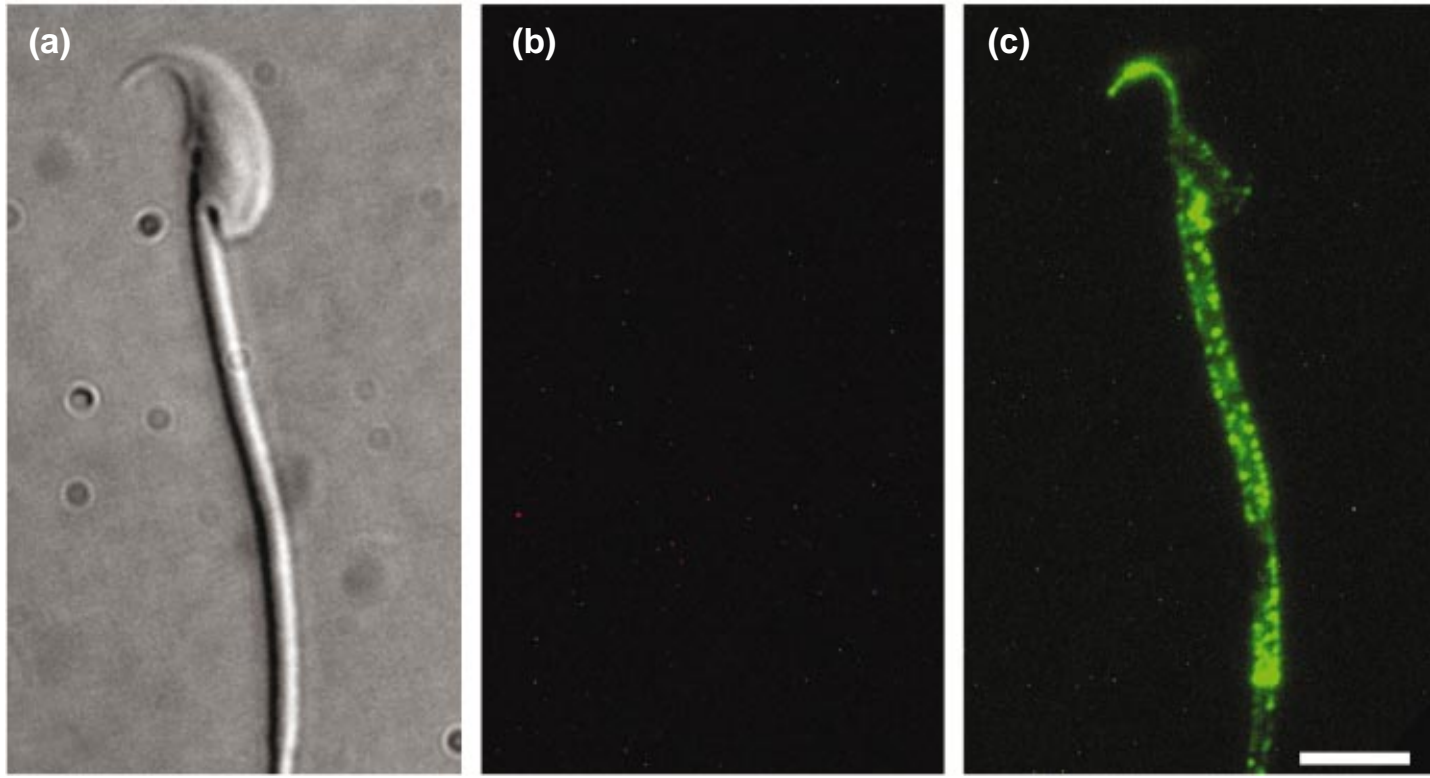

Fig. 4. Photographs showing the presence of basigin on acrosome-reacted mouse spermatozoa by indirect immunofluorescence assay. (a) Differential interference contrast image. (b) Indirect immunofluorescence assay image for intra-acrosomal antigen, acrin 1 (not stained), which was obtained using red channel. (c) Indirect immunofluorescence assay image for basigin, which was obtained using green channel. Scale bar represents $3.2 \mu \mathrm{m}$.

\section{Molecular mass of bsg in the testis and caput, corpus and cauda epididymides}

Anti-bsg antibody recognized a band of $37 \mathrm{kDa}$ in the testis, three bands each of of 37,29 and $27 \mathrm{kDa}$ in the caput epididymides, three bands of 29, 28 and $26 \mathrm{kDa}$ in the corpus epididymides and two bands of 29 and $26 \mathrm{kDa}$ in the cauda epididymides (Fig. 5). These findings indicate a trend in which the molecular size of bsg decreases during epididymal transit.

\section{Effect of anti-bsg antibody on IVF events}

Sperm motility. The anti-bsg antibody had no effect on sperm motility. The average percentage of motile spermatozoa was $67.6 \pm 3.7 \%$ in the presence of anti-bsg antibody compared with $74 \pm 5.2 \%$ in the control groups (TYH only) and $66.3 \pm 7.7 \%$ in the presence of $\mathrm{mMC} 31$.

Primary sperm-zona pellucida binding. In cumulus-free oocytes, 9.2 spermatozoa (on average) were attached to the zona pellucida in the experimental groups that had been treated with anti-bsg antibody compared with 13-15 spermatozoa in the control groups (TYH and mMC31) at $30 \mathrm{~min}$ after insemination.

In the cumulus-invested oocyte group, only 2.9 spermatozoa (on average) could attach to the zona pellucida in the experimental groups treated with anti-bsg antibody, whereas 13.2-14.8 spermatozoa (on average) attached to the zona pellucida at $1 \mathrm{~h}$ after insemination in the control groups

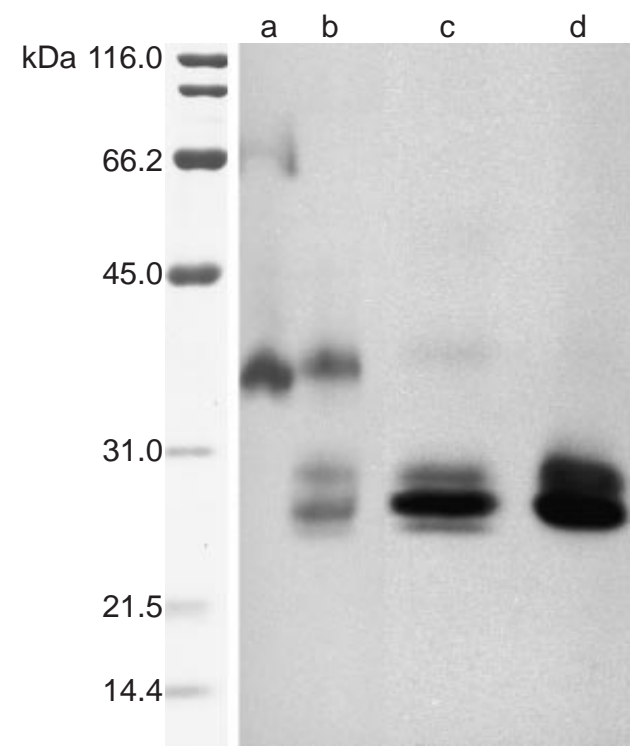

Fig. 5. Immunoblot showing changes in molecular size of basigin in testicular and epididymal spermatozoa in mice. Lane a: testis; lane b: caput spermatozoa; lane c: corpus spermatozoa; lane d: cauda spermatozoa. Note reduction of molecular mass from $37 \mathrm{kDa}$ in both testis and caput spermatozoa to $26 \mathrm{kDa}$ in both corpus and cauda spermatozoa.

(TYH and $\mathrm{mMC} 31)$. The difference between the experimental group and the control group was significant $(P<0.001)$ and indicates that anti-bsg antibody inhibits primary binding (Table 1 and Fig. 6a,b). 

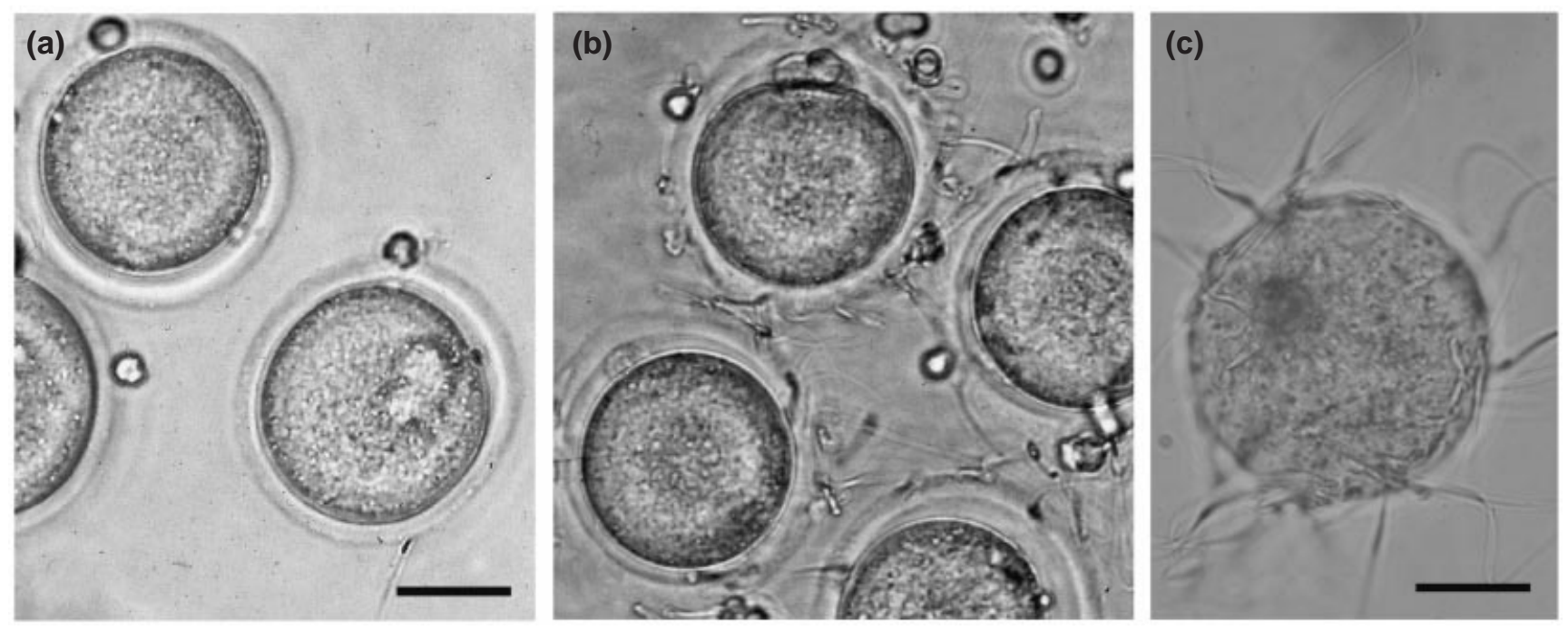

Fig. 6. Photographs showing sperm binding to the mouse zona pellucida. (a) Few spermatozoa are bound to the zona pellucida in the presence of anti-basigin antibody. (b) Control. (c) Spermatozoa are able to bind to the oolemma of the zona-free oocytes in the presence of anti-basigin antibody. Scale bars represent (a,b) $44 \mu \mathrm{m}$ and (c) $35 \mu \mathrm{m}$.

\section{Fertilization rate of the cumulus-invested oocytes with intact zona pellucida}

Pronucleus formation was suppressed in a dosedependent manner at $5 \mathrm{~h}$ after co-incubation of the gametes in the presence of the anti-bsg antibody. The fertilization rate was only $20 \%$ in the anti-bsg antibody groups in comparison with $>70 \%$ in the control groups (TYH and mMC31; Table 2). The difference between the experimental group and the control group was significant $(P<0.001)$.

\section{Sperm-oolemma binding and fertilization of the zona pellucida-free oocytes}

Spermatozoa were able to bind to the zona pellucidafree oocytes (Fig. 6c) equally as well in all the experimental groups treated with anti-bsg and in control groups treated with mMC31 antibody and TYH only. However, the percentage of fertilization was markedly lower in the experimental group treated with anti-bsg antibody (52\%) in comparison with the control groups treated with mMC31 antibody (71\%) and TYH only (76\%). At least 50 oocytes were observed in each group.

\section{Discussion}

The molecular mass of testicular bsg was determined as $37 \mathrm{kDa}$ in the present study. In previous studies the molecular mass of bsg is reported as 43-66 kDa depending on the type of tissue and the level of glycosylation. However, the molecular mass of the core protein is only $27-29 \mathrm{kDa}$ (Nehme et al., 1995; Igakura et al., 1998), indicating that testicular bsg is a highly glycosylated molecule. Findings from the present study show that there is a reduction in the molecular mass of the bsg molecule from $37 \mathrm{kDa}$ in the testis to $29 \mathrm{kDa}$ in the cauda epididymidis during epididymal transit. This result indicates that the bsg molecule undergoes molecular processing and/or deglycosylation during epididymal maturation. The occurrence of deglycosylation during epididymal maturation might have physiological significance.

Spermatozoa are compartmentalized into anterior and posterior head and tail regions. The plasma membrane proteins of spermatozoa are also highly compartmentalized. Sperm plasma membrane proteins undergo a variety of biochemical modifications, such as processing and redistribution, during epididymal maturation (Myles and Primakoff, 1984; Phelps et al., 1990; Cowan et al., 1991; Zhu et al., 2001). Such modifications also occur during capacitation and acrosome reaction (Myles and Primakoff, 1984, 1997; Toshimori, 2000).

As well as biochemical modifications, such as processing, results from the present study demonstrate redistribution of the bsg molecule during epididymal maturation. The bsg molecule was located from the principal piece to the middle piece of spermatozoa. In the present study, there was also a reduction in the molecular mass of bsg during epididymal transit from corpus to cauda epididymides. Changes in localization of bsg appear to be related to epididymal maturation. Localization changes and molecular processing of CE9 (Petruszak et al., 1991) and MC31 (Toshimori, 2000) have been reported in rats during epididymal maturation.

After capacitation, expression of bsg was stronger on the middle piece of spermatozoa. In $12-22 \%$ of capacitated spermatozoa, the head, predominantly at the apical region, and at the anterior acrosome was also stained with anti-bsg 
antibody. These changes in localization might be closely related to the continuing maturation process during capacitation.

Relocation of sperm surface molecules during capacitation and the acrosome reaction has also been reported for some sperm surface molecules, such as PT-1 antigen in guinea-pigs (Myles et al., 1984) and 2B1 (Gaunt et al., 1983; Jones et al., 1990).

The appearance of bsg at the apical region, and at the anterior acrosome of capacitated spermatozoa might be related to the inhibition of sperm-zona pellucida binding and consequently fertilization of the cumulus-invested oocytes with intact zona pellucida. Saling et al. (1979) reported that mouse spermatozoa initially bind to the zona pellucida at the apical ridge over the acrosome under IVF conditions. However, the percentage of fixed capacitated spermatozoa with staining in the head region was only $12 \%$. Although the percentage of staining around the sperm head area was increased two-fold $(22 \%)$ when live capacitated spermatozoa were immunostained under IVF condition, this finding is not sufficient to draw a firm conclusion regarding direct involvement of bsg in sperm-zona pellucida binding. Moreover, the possibility of multivalent interactions of sperm molecules with anti-bsg (polyclonal) antibody cannot be ruled out. Thus, although the involvement of the bsg molecule in sperm-zona pellucida binding remains a possibility, further studies using a monoclonal probe may be helpful to confirm this notion.

The present study also examined whether anti-bsg antibody crossreacts with components of the zona pellucida and possibly inhibits sperm-zona pellucida binding; however, crossreaction with the zona pellucida was not observed in the present study.

The mechanism of sperm-zona pellucida interaction is complex and remains unclear as these steps are thought to be mediated by several sperm molecules, including galactosyltransferase, sp56, zona receptor kinase, spermadhesins, fertilin and zona pellucida components such as ZP3 (for a review, see McLeskey et al., 1998). It is also possible that the anti-bsg antibody acts as an antagonist to some spermatozoa and zona pellucida molecules, which directly facilitate the sperm-zona pellucida binding.

Although anti-bsg antibody markedly inhibited spermzona pellucida binding in the cumulus-intact oocyte group of the present study, it exerted only mild inhibitory effects on sperm-zona pellucida binding in cumulus-free oocytes with intact zona pellucida. This finding indicates that the anti-bsg antibody might affect certain molecular interactions between cumulus-extracellular matrix and sperm surface molecules (bsg), which take place when spermatozoa pass through the cumulus matrix before binding to the zona pellucida.

In the present study, bsg was found at the postacrosomal region and on occasions on the equatorial segment of some acrosome-reacted spermatozoa. Both postacrosomal region and equatorial segment could be involved in sperm-oocyte fusion (Yanagimachi, 1994). Moreover, bsg, EMMPRIN,
CE9 and HT7 are integrin-associated (Berditchevski et al., 1997). Egg integrin has been implicated in sperm-oocyte binding and, in addition, several integrin-associated molecules, such as RDG peptides, fertilin (PH30, ADAM family), inhibit sperm-oocyte binding (for a review, see Evans, 1999). Hence, the present study investigated whether bsg facilitates certain molecular interactions between spermatozoa and oolemma molecules, using anti-bsg antibody. Anti-bsg antibody did not affect sperm-oolemma binding, but only reduced $(52 \%)$ the fertilization rate of the zona pellucida-free oocytes when compared with the control $(70 \%)$. These observations indicate that bsg might help facilitate certain molecular interactions, but that it does not appear to be an essential molecule for sperm-oolemma interaction.

In summary, the bsg molecule was localized on the principal piece of testicular spermatozoa. However, it appeared on the middle piece during epididymal maturation and on the head of spermatozoa after capacitation. These changes in bsg localization might be related to the masking-unmasking phenomena or might be as a result of molecular migration during the maturation process. The maturation process also results in molecular processing, presumably, deglycosylation, consequently reducing the molecular mass of bsg during epididymal transit. Functionally, the bsg molecule that was detected on the sperm head after capacitation might facilitate primary binding and interactions between cumulus-extracellular matrix and sperm surface molecules, for optimization of surface molecules required for sperm-zona pellucida adhesion. Further studies using the direct biochemical affinity assay of the bsg molecule with the zona pellucida and cumulus molecules are required to confirm the role of bsg in sperm-oocyte binding.

The authors are grateful to G. Manandhar, I. Tanii and K. Yoshinaga for their suggestions, Y. Fujii for his excellent technical assistance and to $\mathrm{H}$. Kiyotake for the word processing. This study was supported by grants from JSPS to D. K. Saxena, and the Ministry of Education, Science, Sports and Culture of Japan; grantin-aid for scientific research to K. Toshimori (09670021, 1200338 and 12670022).

\section{References}

Berditchevski F, Chang S, Bodorova J and Hemler ME (1997) Generation of monoclonal antibodies to integrin-associated proteins. Evidence that $\alpha 1$ complexes with emmprin/basigin/OX47/M6 Journal of Biological Chemistry 27229 174-29 180

Biswas C, Zhang Y, DeCastro R, Guo H, Nakamura T, Kataoka H and Nabeshima K (1995) The human tumor cell-derived collagenase stimulatory factor (renamed EMMPRIN) is a member of the immunoglobulin superfamily Cancer Research 55 434-439

Cowan AE, Myles DG and Koppel DE (1991) Migration of the guinea-pig sperm membrane protein $\mathrm{PH}-20$ from one localized surface domain to another does not occur by a simple diffusion-trapping mechanism Developmental Biology 144 189-198

Evans JP (1999) Sperm disintegrins, egg integrins and other cell adhesion molecules of mammalian gamete plasma membrane interactions Frontiers of Bioscience 4 114-131 
Fadool JM and Linser PJ (1993) Differential glycosylation of the 5A11/HT7 antigen by neural retina and epithelial tissues in the chicken Journal of Neurochemistry 60 1354-1364

Fan Q-W, Kadomatsu K, Uchimura K and Muramatsu T (1998) Embigin/basigin subgroup of the immunoglobulin superfamily: different modes of expression during mouse embryogenesis and correlated expression with carbohydrate antigenic markers Development, Growth and Differentiation 40 277-286

Gaunt SJ, Brown CR and Jones R (1983) Identification of mobile and fixed antigens on the plasma membrane of rat spermatozoa using monoclonal antibodies Experimental Cell Research 144 275-284

Igakura T, Kadomatsu K, Kaname T et al. (1998) A null mutation in basigin, an immunoglobulin superfamily member, indicates its important roles in peri-implantation development and spermatogenesis Developmental Biology 194 152-165

Jones R, Shalgi R, Hoyland J and Phillips DM (1990) Topographical rearrangement of a plasma membrane antigen during capacitation of rat spermatozoa in vitro. Developmental Biology 139 349-362

Kasinrerk W, Fiebiger E, Stefanova I, Baumruker T, Knapp W and Stockinger H (1992) Human leukocyte activation antigen M6, a member of the Ig superfamily, is the species homologue of rat OX-47, mouse basigin, and chicken HT7 molecule Journal of Immunology 149 847-854

Kuno N, Kadomatsu K, Fan Q-W, Hagihara M, Senda T, Mizutani S and Muramatsu T (1998) Female sterility in mice lacking the basigin gene, which encodes a transmembrane glycoprotein belonging to the immunoglobulin superfamily FEBS Letters 425 191-194

Laemmli UK (1970) Cleavage of structural proteins during the assembly of the head of bacteriophage T4 Nature 227 680-685

McLeskey SB, Dowds C, Carballada R, White RR and Saling PM (1998) Molecules involved in mammalian sperm-egg interaction International Review of Cytology 177 57-113

Mastroianni L and Manson WA (1963) Collection of monkey semen by electroejaculation Proceedings of the Society for Experimental Biology and Medicine 112 1025-1027

Miyauchi T, Kanekura T, Yamaoka A, Ozawa M, Miyazawa S and Muramatsu T (1990) Basigin, a new, broadly distributed member of the immunoglobulin superfamily, has strong homology with both the immunoglobulin $\mathrm{V}$ domain and the beta-chain of major histocompatibility complex class II antigen Journal of Biochemistry 107 316-323

Miyauchi T, Masuzawa Y and Muramatsu T (1991) The basigin group of the immunoglobulin superfamily: complete conservation of a segment in and around transmembrane domains of human and mouse basigin and chicken HT7 antigen Journal of Biochemistry 110 770-774

Myles DG and Primakoff P (1984) Localized surface antigens of guinea-pig sperm migrate to new regions prior to fertilization Journal of Cell Biology 99 1634-1641

Myles DG and Primakoff P (1997) Why did the sperm cross the cumulus? To get to the oocyte. Functions of the sperm surface proteins $\mathrm{PH}-20$ and fertilin in arriving at, and fusing with, the egg Biology of Reproduction $56320-327$

Myles DG, Primakoff P and Koppel DE (1984) A localized surface protein of guinea-pig sperm exhibits free diffusion in its domain Journal of Cell Biology 98 1905-1909

Nehme CL, Cesario MM, Myles DG, Koppel DE and Bartles JR (1993) Breaching the diffusion barrier that compartmentalizes the transmembrane glycoprotein CE9 to the posterior-tail plasma membrane domain of the rat spermatozoon Journal of Cell Biology 120 687-694

Nehme CL, Fayos BE and Bartles JR (1995) Distribution of the integral plasma membrane glycoprotein CE9 (MRC OX-47) among rat tissues and its induction by diverse stimuli of metabolic activation Biochemical Journal 310 693-698

Petruszak JAM, Nehme CL and Bartles JR (1991) Endoproteolytic cleavage in the extracellular domain of the integral plasma membrane protein CE9 precedes its redistribution from the posterior to the anterior tail of the rat spermatozoon during epididymal maturation Journal of Cell Biology 114 917-927

Phelps BM, Koppel DE, Primakoff P and Myles DG (1990) Evidence that proteolysis of the surface is an initial step in the mechanism of formation of sperm cell surface domains Journal of Cell Biology 111 1839-1847

Saling PM, Sowinski J and Storey BT (1979) An ultrastructural study of epididymal mouse spermatozoa binding to zonae pellucidae in vitro: sequential relationship to the acrosome reaction Journal of Experimental Zoology 209 229-238

Saxena DK, Tanii I, Yoshinaga K and Toshimori K (1999) Role of intraacrosomal antigenic molecules acrin 1 (MN7) and acrin 2 (MC41) in penetration of the zona pellucida in fertilization in mice Journal of Reproduction and Fertility 117 17-25

Toshimori K (2000) Sperm plasma membrane modifications associated with fertilization in mammals Journal of Reproduction and Development $\mathbf{4 6}$ 65-78

Toshimori K, Tanii I, Araki S and Oura C (1992) A rat sperm flagellar surface antigen that originates in the testis and is expressed on the flagellar surface during epididymal transit Molecular Reproduction and Development 32 399-408

Toshimori K, Saxena DK, Tanii I and Yoshinaga K (1998) An MN9 antigenic molecule, equatorin, is required for successful sperm-oocyte fusion in mice Biology of Reproduction 59 22-29

Towbin H, Staehelin T and Gordon J (1979) Electrophoretic transfer of proteins from polyacrylamide gels to nitrocellulose sheets: procedure and some applications Proceedings National Academy of Sciences USA $64350-4354$

Toyoda Y, Yokoyama M and Hoshi T (1971) Studies on the fertilization of mouse eggs in vitro. Japanese Journal of Animal Reproduction 16 $147-157$

Wakayama T, Nagata K, Ohashi K, Mizuno K, Tanii I, Yoshinaga K, Oh-Oka T and Toshimori K (2000) The expression and cellular localization of the sperm flagellar protein MC31/CE9 in the rat testis: possible posttranscriptional regulation during rat spermiogenesis Archives of Histology and Cytology 63 33-41

Yanagimachi R (1994) Mammalian fertilization. In The Physiology of Reproduction, Vol. 2 pp 189-317 Eds E Knobil and JD Neill. Raven Press, New York

Yoshida S, Shibata M, Yamamoto S, Hagihara M, Asai N, Takahashi M, Mizutani S, Muramatsu T and Kadomatsu K (2000) Homo-oligomer formation by basigin, an immunoglobulin superfamily member, via its $\mathrm{N}$-terminal immunoglobulin domain European Journal of Biochemistry $2674372-4380$

Zhu GZ, Myles DG and Primakoff P (2001) Testase 1 (ADAM 24) a plasma membrane-anchored sperm protease implicated in sperm function during epididymal maturation or fertilization Journal of Cell Science 114 $1787-1794$

Received 6 August 2001.

First decision 8 October 2001.

Revised manuscript received 12 November 2001.

Accepted 29 November 2001. 